\title{
Ventriculo-arterial Coupling and the Areas under the End-systolic Pressure-volume Relation
}

\author{
Rachad M. SHoucri, PhD
}

\begin{abstract}
SUMmary
Ventriculo-arterial coupling is expressed as the ratio $E_{\mathrm{max}} / e_{\mathrm{am}}$ (maximum ventricular elastance/arterial elastance). Different areas under the end-systolic pressure-volume relation (ESPVR) are expressed in terms of $E_{\max } / e_{\mathrm{am}}$. The explicit inclusion of the active force of the myocardium in the mathematical formalism describing the pressure-volume relation (PVR) leads to new insight into the mechanics of left ventricular contraction. Applications to experimental data rclated to stroke work area $S W$ under ESPVR are discussed and provide further evidence for the consistency of the mathematical formalism used. (Jpn Héart J 1997; 38: 253-262)
\end{abstract}

Key words: End-systolic pressure-volume relation, Active force of the myocardium, Maximum elastance $E_{\max }$, Mechanics of left ventricular contraction, Ventriculo-arterial coupling

7 HERE has been some interest recently in studying ventriculo-arterial coupling, expressed by the ratio $E_{\max } / e_{\mathrm{am}}$ (maximum elastance/corresponding arterial elastance). ${ }^{1-4)}$ The purpose of the present study is to review some basic equations used in the study of the end-systolic pressure-volume relation (ESPVR) with the aim of emphasizing the following concepts:

a) The mathematical formalism used to describe the pressure-volume relation (PVR) explicitly includes the active force of the myocardium.

b) Stroke work $(S W)$, as well as other areas under ESPVR are used to express ventriculo-arterial coupling as a function of $E_{\max } / e_{\mathrm{am}}$ (note that the corresponding notation $E_{\mathrm{es}} / E_{\mathrm{a}}$ is also used). ${ }^{1-4)}$ Clinical applications of different areas under ESPVR as well as their significance for the study of left ventricular adaption to load have been discussed. ${ }^{12,13}$ The following discussion stresses the point of view that the study of the mechanics of left ventricular contraction should be based on an integrated approach to the interrelation between different areas under ESPVR (Figures 3 and 4) rather than an isolated study of the stroke work area $S W$.

\footnotetext{
From the Department of Mathematics and Computer Science, Royal Military College of Canada, Kingston, Ontario, Canada.

Address for correspondence: Rachad M. Shoucri, PhD, Department of Mathematics and Computer Science, Royal Military College of Canada, Kingston, Ontario, Canada K7K, 5L0.

Received for publication August 24, 1996.

Accepted November 13, 1996
} 
Table I. Experimental Verification of Equations (7) and (8)

\begin{tabular}{|c|c|c|c|c|c|c|c|c|c|c|c|}
\hline $\begin{array}{l}V_{\text {cet }} \\
\mathrm{m} l\end{array}$ & $\begin{array}{l}V_{\mathrm{m}} \\
\mathrm{m} l\end{array}$ & $\underset{\mathrm{mmHg} / \mathrm{m} l}{E_{\max }}$ & $E_{\text {max }} / e_{\text {am }}$ & $\begin{array}{c}M \\
\mathrm{mmHg}\end{array}$ & $\begin{array}{c}V_{\mathbf{1}} \\
\mathrm{m} l\end{array}$ & $\begin{array}{l}V_{100} \\
\mathrm{~m} l\end{array}$ & $\begin{array}{l}V_{\mathrm{dm}} \\
\mathrm{m} l\end{array}$ & $\begin{array}{c}M \times \\
\left(V_{\mathrm{cd}}-V_{\mathrm{r}}\right)\end{array}$ & $\begin{array}{c}S W \\
\mathrm{Eq} \cdot(7)\end{array}$ & $\begin{array}{c}M / \\
\left(V_{\mathrm{cd}}-V_{\mathrm{l}}\right)\end{array}$ & $\begin{array}{c}2 \mathrm{~m} \\
\mathrm{Eq} .(8)\end{array}$ \\
\hline 55.4 & 35.2 & 7.2 & 0.96 & 84.6 & 31.8 & 33.6 & 19.7 & 1997 & 2293 & 3.6 & 3.6 \\
\hline 49.3 & 35.9 & 9.0 & 0.91 & 82,0 & 30.4 & 34.2 & 23.1 & 1550 & 1541 & 4.3 & 4.5 \\
\hline 54.9 & 41.1 & 7.5 & 0.61 & 77.0 & 30.6 & 34.5 & 21.2 & 1871 & 2004 & 3.2 & 3.5 \\
\hline 58.2 & 45.6 & 6.9 & 0.48 & 75.0 & 30.4 & 33.2 & 18.7 & 2085 & 2359 & 2.7 & 3.0 \\
\hline 58.4 & 45.6 & 6.8 & 0.43 & 61.9 & 29.8 & 32.3 & 17.6 & 1770 & 2380 & 2.2 & 2.9 \\
\hline 41.9 & 31.4 & 10.7 & 1.27 & 71.7 & 31.6 & 35.5 & 26.2 & 739 & 650 & 6.9 & 5.3 \\
\hline 49.5 & 34.0 & 13.1 & 1.33 & 106.0 & 28.6 & 31.0 & 23.4 & 2215 & 2186 & 5.1 & 6.4 \\
\hline 51.0 & 35.8 & 11.0 & 1.04 & 102.3 & 30.2 & 30.7 & 21.6 & 2128 & 2376 & 4.9 & 5.5 \\
\hline 53.8 & 37.8 & 11.7 & 0.89 & 104.8 & 27.2 & 29.0 & 20.5 & 2788 & 3233 & 3.9 & 5.8 \\
\hline 55.5 & 39.8 & 10.5 & 0.76 & 105.8 & 28.1 & 29.0 & 19.5 & 2899 & 3339 & 3.9 & 5.2 \\
\hline \multirow[t]{3}{*}{43.6} & 31.3 & 15.0 & 1.93 & 94.4 & 33.0 & 34.8 & 28.1 & 1001 & 810 & 8.9 & 6.7 \\
\hline & & & & & & & mean & 1913 & 2106 & 4.5 & 4.8 \\
\hline & & & & & & & SD & 654 & 847 & 1.9 & 1.3 \\
\hline
\end{tabular}

$V_{\mathrm{cd}}=$ end-diastolic left ventricular volume; $V_{\mathrm{m}} \approx V_{\mathrm{cs}}=$ end-systolic left ventricular volume; $E_{\mathrm{max}}=\operatorname{maximum}$ elastance, $\ell_{\mathrm{am}}=$ arterial elastance; $M\left(V_{\mathrm{ed}}-V_{\mathrm{l}}\right)=$ see eq. (9), $V_{100}=V_{\mathrm{dm}}+100 / E_{\mathrm{max}}$ values for $V_{\mathrm{ed}}, V_{\mathrm{m}}, E_{\max }, E_{\mathrm{max}} /$ $\mathrm{e}_{\mathrm{an}}, M, V_{\mathrm{I}}, V_{100}$ are taken from Table $\mathrm{I}$ of Little and Cheng ${ }^{3}$.

c) Following experimental results presented by Burkhoff and Sagawa, Asanoi et $\mathrm{al}_{1}{ }^{6}$ Fourie et $\mathrm{al}^{7}{ }^{7}$ the present study takes the position that under normal physiological conditions the left ventricle operates near maximum oxygen efficiency with $E_{\max } / e_{\mathrm{am}} \approx 2, E_{\max } / e_{\mathrm{am}} \approx 1$ (maximum $S W$ ) corresponds to a mildly depressed state of the heart while $E_{\max } / e_{\mathrm{am}} \ll 1$ corresponds to a severely depressed state. Based on experimental observations it has been reported by Burkhoff and Sagawa $a^{5)}$ that "... it does not seem possible to obtain simultaneously a physiological SV (stroke volume), end-diastolic volume, and end-systolic pressure with the condition $E_{\mathrm{a}}=E_{\mathrm{es}}$. $E_{\mathrm{a}}$ must always be smaller than $E_{\mathrm{es}}$ to obtain physiological conditions...".

d) New applications to experimental data related to $S W$ taken from Little and Cheng) are given in Table I and applications to simulation data related to $S W$ taken from Takaoka et al ${ }^{1)}$ are presented in Table II. The factor A introduced in Eq. (10) of Takaoka et al ${ }^{1)}$ is derived again, and it is shown that $A=(1 /$ 2) $(S W / T W$ ), where $S W=$ stroke work and $T W=$ total area under ESPVR (Figure 3). These results provide further evidence for the consistency of the mathematical formalism used in the present study.

e) The concept of "preload recruitable stroke work" is reviewed. It is suggested that the apparent linearity between $S W$ and the end-dialostic volume $V_{\text {ed }}$ is only an approximation.

Although the ESPVR is slightly curvilinear, the linear model shown in Figure 2 is adopted. This model will simplify greatly the mathematical formalism to be developed, without affecting the basic concepts to be discussed. 


\section{Mathematical Method}

The cross-section of a three-dimensional cavity of the left ventricle is shown in Figure 1. The helical structure of the fibre generates a radial active force/unit volume D, as explained by Shoucri. ${ }^{8,9}$ In a quasi-static approximation (inertia and viscous forces neglected), the equilibrium of forces in the radial direction is given by ${ }^{8,9)}$

$$
\bar{D} h-P=E\left(V_{e d}-V\right)
$$

where $\bar{D} h=\int_{a}^{b} D d r=$ radial active force/unit area developed on the inner surface of the myocardium

$h=b-a=$ thickness of myocardium

$a=$ inner radius of the myocardium

$b=$ outer radius of the myocardium

$E=$ left ventricular elastance, slope of the P-V line in Figure 2

$P=$ left ventricular pressure

$V=$ left ventricular volume

$V_{\text {ed }}=$ end-diastolic ventricular volume (when $d V / d t=0$ )

Near end-systole when $E$ reaches its maximum value $E_{\max }$, a suffix $\mathrm{m}$ is added to the variables

$$
(\bar{D} h)_{\mathrm{m}}-P_{\mathrm{m}}=E_{\max }\left(V_{\mathrm{ed}}-V_{\mathrm{m}}\right)
$$

Eq. (1a) can be separated into two equations

$$
\begin{gathered}
P=E\left(V-V_{\mathrm{d}}\right) \\
\bar{D} h=E\left(V_{\mathrm{ed}}-V_{\mathrm{d}}\right)
\end{gathered}
$$

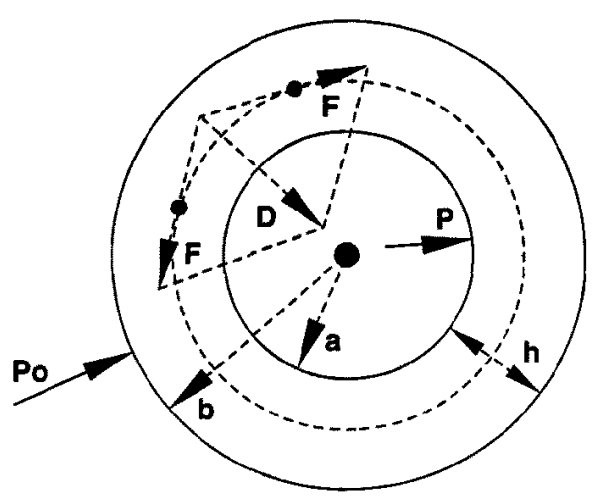

Figure 1. Simplified cross-section of the left-ventricle, the helical muscular fibre of the myocardium is projected as a circle. $\mathrm{D}$ is the radial active force/unit volume of myocardium. $h=b-a$ is the thickness of myocardium, $a=$ inner radius, $b=$ outer radius, $P=$ ventricular pressure, $P_{\mathrm{o}}=$ outer pressure assumed zero. 


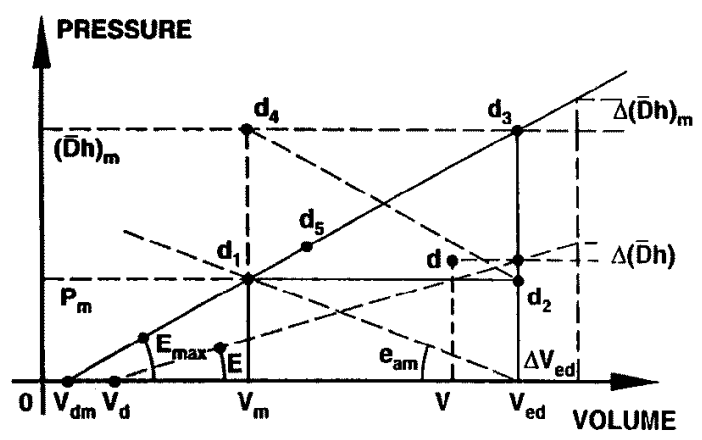

Figure 2. Simplified pressure volume relation (PVR), slope $E=$ ventricular elastance, slope $e_{2 \mathrm{~m}}=$ arterial elastance when $E=E_{\max }$. During an ejecting contraction, PVR is represented by the loop $V_{c \mathrm{~d}} d_{2} d_{1} V_{\mathrm{m}}$, the systolic pressure $P_{\mathrm{rn}}$ is assumed constant. Note the changes $\Delta(\bar{D} h), \Delta(\bar{D} h)_{\mathrm{m}}$ corresponding to $\Delta V_{\mathrm{ed}}$ as an expression of the FrankStarling mechanism. $d_{5}$ is the middle point of the line $d_{3} V_{\mathrm{dm}}$ (ESPVR).

Near end-systole, Eq. (1b) can also be separated as follows

$$
\begin{gathered}
P_{\mathrm{m}}=E_{\max }\left(V_{\mathrm{m}}-V_{\mathrm{dm}}\right) \\
(\bar{D} h)_{\mathrm{m}}=E_{\max }\left(V_{\mathrm{cd}}-V_{\mathrm{dm}}\right)
\end{gathered}
$$

The intersection $V_{\mathrm{d}}$ or $V_{\mathrm{dm}}$ of the PVR with volume axis is shown in Figure 2. If $E$ (or $E_{\text {max }}$ ) is kept constant, and $V_{\mathrm{d}}$ (or $V_{d m}$ ) kept constant, Eq. (2b) and (3b) give

$$
\begin{gathered}
\Delta(\bar{D} h)=E \Delta V_{\mathrm{ed}} \\
\Delta(\bar{D} h)=E_{\mathrm{max}} \Delta V_{\mathrm{ed}}
\end{gathered}
$$

The variations $\Delta(\bar{D} h), \Delta(\bar{D} h)_{\mathrm{m}}$ and $\Delta V_{\text {ed }}$ shown in Figure 2 represent another way to express the Frank-Starling mechanism. Eq. (1-4) together with Figure 2 summarize the basic mechanism of cardiac contraction. Experimental verification of Eq. (1-4) has been extensively discussed ${ }^{8-13)}$ and thus will not be repeated here. In this study the focus is rather on the physiological insight that can be gained from an integrated study of the areas under ESPVR, as well as some further experimental evidence of the consistency of the mathematical formalism derived by Shoucri. ${ }^{11,12)}$

\section{Results}

Mechanics of left ventricular contraction: It is assumed below that during systolic contraction $P \approx P_{\mathrm{m}}$ is nearly constant, and that when $E=E_{\max }$ the left ventricular volume $V_{\mathrm{m}} \approx V_{\mathrm{es}}\left(V_{\mathrm{es}}=\right.$ end-systolic volume when $\left.d V / d t=0\right)$. During normal systolic ejection, the point $\mathrm{d}$ of coordinates $\left(\bar{D} h, V\right.$ moves from point $d_{2}$ to 


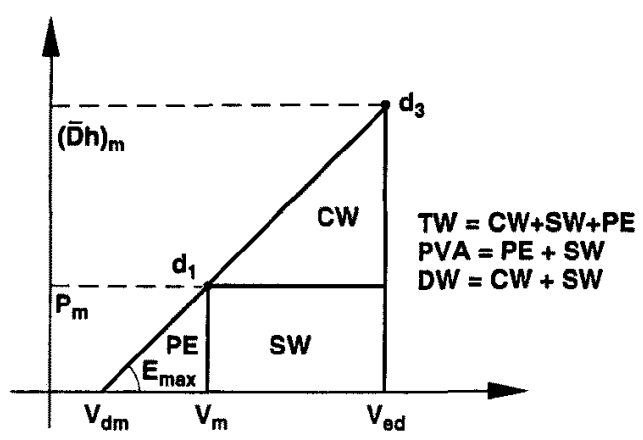

Figure 3. Areas under ESPVR. $P E=$ potential energy absorbed by the internal metabolism of myocardial contraction, $S W=$ stroke work delivered to systemic circulam tion, $C W=$ contraction work absorbed by the passive medium of myocardium.

point $d_{4}$ corresponding to $(\bar{D} h)_{\mathrm{m}}$ (Figure 2). In a quasi-static approximation (inertia and viscous forces neglected), the maximum active force $(D h)_{\mathrm{m}}$ is equal to the maximum isovolumic pressure $P_{\text {isom }}$ represented by point $d_{3}$ in Figure 2, and in general $(\bar{D} h)$ is equal to $P_{\text {iso }}$ by the symmetry of the lines $d_{2} d_{4}$ and $d_{1} d_{3}$. Diffcrent notations have been used for the active force $(\bar{D} h)$ (ejecting contraction), and the isovolumic pressure $P_{\text {iso }}$ (non-ejecting contraction) to emphasize that $\bar{D} h$ is a force and has magnitude and direction, whereas $P_{\text {iso }}$ is a scalar quantity.

An immediate and interesting result that can be deduced from Figures 2 and 3 is that when $d_{1}$ moves along the ESPVR $d_{3} V_{\mathrm{dm}}$, the stroke work $S W$ represented by the area $V_{\text {ed }} d_{2} d_{1} V_{\mathrm{m}}$ is maximum when $d_{1}$ coincides with $d_{5}$ (midpoint of the segment $d_{3} V_{\mathrm{dm}}$ ) [note that $d_{1}$ and $d_{5}$ coincides when $\left.E_{\max } / e_{\mathrm{am}} \approx 1\right]$. The condition $E_{\max } / e_{\text {arm }} \approx 2$ (maximum oxygen efficiency) corresponds to $d_{1}$ below $d_{5}$ on the line $d_{3} V_{\mathrm{dm}}$, and the condition $E_{\max } / e_{\mathrm{am}} \ll 1$ corresponds to $d_{1}$ above $d_{5}$. It is seen that when $E_{\max } / e_{\mathrm{am}}<1, S W$ decreases when $P_{\mathrm{m}}$ increases, creating cardiac insufficiency. Maximum oxygen efficiency as the normal physiological state of the myocardium has also been suggested by Burkhoff and Sagawa ${ }^{5)}$, Asanoi et a ${ }^{60}$, Fourie et $\mathrm{al}^{7}$ ) and Elzinga and Westerhof. ${ }^{15)}$ One can consequently see how the introduction of the active force $\bar{D} h$ in the formalism describing PVR has led to a simple and consistent description of some aspects of the mechanism of left ventricular contraction as summarized by the different areas under ESPVR in Figures 2 and 3 . The application of these areas to the study of physiological adaption of the left ventricle to change of load as well as different clinical applications has been discussed ${ }^{12,13)}$. Some aspects of the relation between $S W$ and $E_{\max } / e_{\mathrm{am}}$ are discussed below, as are possible relations with the areas $C W$ and $P E$.

Experimental verification: The experimental application to be discussed first in this section is the relation between stroke work $S W$ and $V_{\text {ed. }}$. From Figure 3 and from Shoucri, ${ }^{11,12)}$ we obtain 


$$
\frac{S W}{T W}=2 \frac{E_{\mathrm{max}} / e_{\mathrm{am}}}{\left(1+E_{\max } / e_{\mathrm{am}}\right)^{2}}
$$

where $T W$ is the total area under ESPVR. When $d_{1}$ moves along the line $d_{3} V_{\mathrm{dm}}$ (Figure 2), $S W$ is maximum and equals $(S W)_{\max }=T W / 2$ when $d_{1}$ coincides with $d_{5}$ ( $d_{5}$ is the middle point of the segment $d_{3} V_{\mathrm{dm}}$ ). In this case $E_{\mathrm{max}} / e_{\mathrm{am}} \approx 1$ and Figure 2 provides direct and simple proof of this result. The fact that $(S W)_{\max }$ corresponds to $E_{\max } / e_{\mathrm{am}} \approx 1$ has been experimentally observed by several authors (Asanoi et al, ${ }^{6)}$ Burkhoff and Sagawa ${ }^{5)}$ Sunagawa et al $^{18)}$ and Little and Cheng ${ }^{4}$ ). Equation (5) is equivalent to Eq. (7) of Little and Cheng ${ }^{4)}$ and Eq. (2) of Little and Cheng. ${ }^{3)}$ There is a difference of a factor of 2 because normalization in Eq. (5) with respect to $\mathcal{T W}$, while Little and Cheng normalized with respect to $(S W)_{\max }=\mathcal{T} W / 2$. By noting that the total area $T W$ under ESPVR is given by

$$
T W=\frac{1}{2}(\bar{D} h)_{m}\left(V_{\mathrm{ed}}-V_{\mathrm{dm}}\right)
$$

Eq. (3b), (5) and (6) give

$$
S W=m\left(V_{\mathrm{ed}}-V_{\mathrm{dm}}\right)^{2}
$$

with

$$
m=E_{\max } \frac{E_{\max } / e_{\mathrm{am}}}{\left(1+E_{\max } / e_{\mathrm{am}}\right)^{2}}
$$

Eq. (7) is quadratic in $V_{\mathrm{ed}}-V_{\mathrm{dm}}$, and has been compared in Table $\mathrm{I}$ to the linear formula

$$
S W_{\mathrm{I}}=M\left(V_{\mathrm{ed}}-V_{\mathrm{I}}\right)
$$

given by Little and Cheng ${ }^{3,4)}$ where $M$ is preload recruitable stroke work (Glower et $\mathrm{al}^{17)}$ ) and $V_{\mathrm{I}}$ the axis intercept of the linear Eq. (9). The intercept $V_{\mathrm{dm}}$ of ESPVR (see Figure 2) has been calculated from the formula $V_{100}=V_{\mathrm{dm}}+100 / E_{\max }$ given by Eq. (2) of Little and Cheng. ${ }^{3)}$ The comparison between $S W_{1}$ (linear model) and $S W($ Eq. 7) in Table I is good. Note that data taken from Table I of Little and Cheng $^{3)}$ represent averages for experimental results for 8 animals and not data from a single experiment, so some fluctuation is expected. Since $S W_{\mathrm{I}}=\left\langle M /\left(V_{\text {ed }}\right.\right.$ $\left.-V_{\mathrm{I}}\right)\left(V_{\mathrm{ed}}-V_{\mathrm{l}}\right)^{2}$ the values of $M /\left(V_{\mathrm{ed}}-V_{\mathrm{l}}\right)$ have also been compared in Table I with the values of $2 \mathrm{~m}(\mathrm{Eq} .8)$. This comparison was also good, and factor 2 accounts for the fact that the slopes of a quadratic curve and a linear curve differ by a factor of 2. Statistical t-test of the means shown in Table I indicates that the difference is not statistically significant. The linear model (Eq. 9) appears to be an approximation and does not seem to reflect a basic characteristic of left ventricu- 
lar contraction.

We shall now discuss the derivation of the quantity A introduced in Eq. (10) of Takaoka et al. ${ }^{1)}$

A simplc manipulation of Eq. (3b) and (6)-(7) gives

$$
S W=E_{\max }\left(V_{\mathrm{ed}}-V_{\mathrm{dm}}\right)^{2}\left(E_{\max } / e_{\mathrm{am}}\right) /\left(1+E_{\max } / e_{\mathrm{am}}\right)^{2}
$$

Eq. (10) has been derived differently by Burkhoff and Sagawa ${ }^{5)}$ and Sunagawa et al. ${ }^{18)}$ One also has

$$
S W=P_{\mathrm{m}}\left(V_{\mathrm{ed}}-V_{\mathrm{m}}\right)=E_{\max }\left(V_{\mathrm{m}}-V_{\mathrm{dm}}\right)\left(V_{\mathrm{ed}}-V_{\mathrm{m}}\right)
$$

By making $k=e_{a \mathrm{am}} / E_{\max }$ and by equating Eq. (10)-(11) we obtain, using Eq. $(6)-(7)$

$$
k /(1+k)^{2}=(1 / 2)(S W / T W)=A
$$

The dimensionless quantity A is given in Eq. (10) of Takaoka et $\mathrm{al}^{1)}$ and was used by them to calculate $e_{\mathrm{am}}$ (or $E_{\mathrm{a}}$ ), but its relation to the curve for $S W / T W$ given in Figure 4 is not derived. One should note how the mathematical model used in the present study allows a simple derivation of an expression for A. Moreover, by writing $K=1 / k$ we obtain $S W / T W=2 K /(1+K)^{2}$. Derivation with respect to $K$ gives $(d / d K)(S W / T W)=2 /(1+K)^{2}-4 K /(1+K)^{3}$, which is zero when $K=1$, or $E_{\max } / e_{a m}=1$. This corresponds to the maximum of the curve $S W / T W$ in Figure 4. For $K=1,(S W)_{\max }=T W / 2$, which is the maximum value of the stroke volume $\left(d_{1}\right.$ and $d_{5}$ in Figures 2 and 3$)$.

A further validation of Eq. (7) and (8) is provided by the results of Table II. The values of $V_{\mathrm{dm}}, E_{\max } / e_{\mathrm{am}}$ and $V_{\mathrm{ed}}$ are taken from Figure 6A of Takaoka et al ${ }^{1)}$ (with an obvious change in notation, for instance Takaoka et al ${ }^{\text {) }}$ use $E_{\mathrm{a}} / E_{\max }$

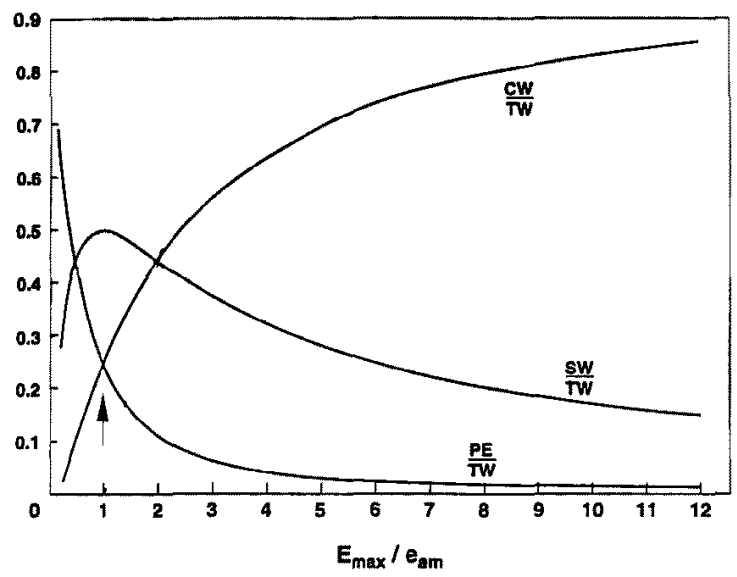

Figure 4. Relations between $S W / T W, C W / T W, P E / T W$, and $E_{\text {max }} / e_{\text {am }}$. 
Table II. Verification of Equations (7) and (8) from Simulation Data

\begin{tabular}{|c|c|c|c|c|c|}
\hline $\begin{array}{l}V_{\mathrm{dm}} \\
\mathrm{m} l\end{array}$ & $\frac{E_{\max }}{\mathrm{mmHg} / \mathrm{m} l}$ & $E_{\mathrm{max}} / \ell_{\mathrm{am}}$ & $\stackrel{m}{\mathrm{mmHg} / \mathrm{ml} l \mathrm{Eq} .(8)}$ & $\begin{array}{l}V_{e d} \\
\mathrm{~m} l\end{array}$ & $\begin{array}{c}\mathrm{SW} \\
\mathrm{mmHg} / \mathrm{m} l \mathrm{Eq} .(7)\end{array}$ \\
\hline & & & & 20 & 222 \\
\hline \multirow[t]{3}{*}{7} & 7 & $1 / 3$ & 1.313 & 30 & 695 \\
\hline & & & & 40 & 1430 \\
\hline & & & & 20 & 263 \\
\hline \multirow[t]{3}{*}{7} & 7 & $1 / 2$ & 1.556 & 30 & 823 \\
\hline & & & & 40 & 1695 \\
\hline & & & & 20 & 296 \\
\hline \multirow[t]{3}{*}{7} & 7 & 1 & 1.750 & 30 & 926 \\
\hline & & & & 40 & 1906 \\
\hline & & & & 20 & 263 \\
\hline \multirow[t]{3}{*}{7} & 7 & 2 & 1.556 & 30 & 823 \\
\hline & & & & 40 & 1695 \\
\hline & & & & 20 & 222 \\
\hline \multirow[t]{2}{*}{7} & 7 & 3 & 1.313 & 30 & 695 \\
\hline & & & & 40 & 1430 \\
\hline
\end{tabular}

$V_{\mathrm{dm}}=$ intercept of ESPVR with volume axis; $V_{\mathrm{ed}}=$ end-diastolic volume; $E_{\max }=$ maximum slope of ESPVR; $e_{\mathrm{am}}=$ corresponding arterial elastance. Values for $V_{\mathrm{dm}}, V_{\mathrm{ed}}, E_{\max }, E_{\max } / e_{\mathrm{atn}}$ are taken from Figure 6A of Takaoka et ally ${ }^{1 /}$ with an obvious change in the notation.

while the ratio $E_{\max } / e_{a m}$ is used in the present study). In Table II, $\mathrm{m}$ is calculated using Eq. (8) and $S W$ using Eq. (7). The values obtained for $S W$ compare very well with the corresponding values of $S W$ that can be measured from Figure $6 \mathrm{~A}$ of Takaoka et al. ${ }^{17}$ In particular note the equality of the values of $\mathrm{m}$ for $E_{\max } /$ $e_{\mathrm{am}}=1 / 3$ and 3, or for $E_{\max } / e_{\mathrm{am}}=1 / 2$ and 2, that results by applying Eq. (8), and the equalities of the corresponding values of $S W$ as shown in Table II and verified by the corresponding values of $S W$ in Figure $6 \mathrm{~A}$ of Takaoka et al. ${ }^{11}$ Here again, Eq. (7) and the results of Table II suggest that the linear dependence of $S W$ on $V_{\text {ed }}$ given by Eq. (9) is an approximation.

Other areas appearing under ESPVR are $C W$ and $P E$. $C W$ seems to be related to the energy absorbed in the contraction of myocardium (see appendix $\mathrm{A}$ of Shoucri $\left.{ }^{11}\right)$. The area $P E$ appears to be related to the energy absorbed by the internal metabolism of the heart. For example Takaoka et $\mathrm{al}^{16)}$ observed that the area $P E$ increases in cardiomyopathy cases. Variations of areas $S W / T W, C W /$ $T W, P E / T W$, with $E_{\max } / e_{\text {am }}$ based on previous theoretical relations ${ }^{12)}$ are shown in Figure 4. Experimental verification of these curves can be found in Figure 5 of Shoucri. ${ }^{12)}$ The curve for $S W / T W$ is similar to Figure 2 of Little and Cheng, ${ }^{4)}$ Figure 7 of Fourie et al, ${ }^{7)}$ and Figure 4 of Takaoka et al. ${ }^{1)}$ The interrelation between these different areas comes from the fact that $S W / T W+C W /$ $T W+P E / T W=1$. No experimental investigation of this interrelation has yet been undertaken. 


\section{Conclusion}

Important features of this study are as follows:

a) The inclusion of the radial active force of the myocardium $\bar{D} h$ in the mathematical formalism describing ESPVR;

b) The possibility of using the ratio $E_{\max } / e_{\text {am }}$ to distinguish between normal $\left(E_{\max } / e_{\mathrm{am}} \approx 2\right)$, mildly depressed $\left(E_{\max } / e_{\mathrm{am}} \approx 1\right)$ (maximum $S W$ ), and severely depressed states of the heart $\left(E_{\max } / e_{a m} \ll 1\right)$;

c) It is suggested that the interrelation between different areas under ESPVR (summarized in Figures 3 and 4) plays a fundamental role in understanding cardiac mechanics. The lincar cquation Eq. (9) for $S W_{\mathrm{I}}$ for instance does not represent a fundamental aspect of cardiac contraction, but rather appears simply to be an isolated approximation. Figure 4 shows that there is a need for an integrated approach for the modelling of different areas under ESVPR. For instance in Figure 2, the study of the area $V_{\mathrm{ed}} d_{2} d_{4} V_{\mathrm{m}}$ (or by symmetry $V_{\mathrm{ed}} d_{3} d_{1} V_{\mathrm{m}}$ ) which represents the work of the active force $\bar{D} h$, when $\bar{D} h$ moves from $d_{2}$ to $d_{4}$, has not received any attention.

d) An important aspect of this interrelation between different areas under ESPVR, not discussed in this study, is the relation between oxygen consumption and the different areas of $P E, S W, C W$ under ESPVR. This problem has been discussed ${ }^{11,12)}$ and requires further experimental as well as theoretical study.

The possibility of non-invasive implementation of the results of this study for clinical work using M-mode echocardiography for example is another challenge for experimentalists and has been discussed. ${ }^{12)}$ Finally, it should be mentioned that an interesting review of cardiac mechanics based on PVR has been given by Suga. ${ }^{19}$;

\section{REFERENCES}

1. Takaoka H, Suga H, Goto Y, Hata K, Takeuchi M. Cardiodynamic conditions for the linearity of preload recruitable stroke work. Heart and Vessels 1995; 10: 57-68

2. Nevo E, Marmor M, Lanir Y, Weiss TA, Marmor A. A new methodology for non-invasive clinical assessment of cardiovascular performance and of ventricular-arterial coupling during stress. Heart and Vessels 1995; 10: 24-34.

3. Little WC, Cheng CP. Left ventricular-arterial coupling in conscious dogs. Am J Physiol 1991; 261: $\mathrm{H} 70-\mathrm{H} 76$.

4. Little WC, Cheng CP. Coupling of the left ventricular and arterial system. Med Biol Eng Comput 1994; 32: 205-9.

5. Burkhoff D, Sagawa K. Ventricular efficiency predicted by an analytical model. Am J Physiol 1986; 250: R1021-R1027.

6. Asonoi H, Sasayama S, Kamegama T. Ventriculo arterial coupling in normal and failing heart in humans. Circ Res 1989; 65: 483-93.

7. Fourie PR, Coetzce AR, Bolliger CT. Pulmonary artery compliance: its role in right ventriculararterial coupling. Cardio Res 1992; 26: 839-44. 
8. Shoucri RM. The pressure volume relation and the mechanics of left ventricular contraction. Jpn Heart J 1990; 31: 713-29.

9. Shoucri RM. Theoretical study of pressure-volume relation in left ventricle. Am J Physiol 1991; 260: H282-H29l.

10. Shoucri RM. Non-linear pressure-volume relation in left ventricle. Jpn Heart J 1991; 32:337-46.

11. Shoucri RM. Theoretical study related to left ventricular energetics. Jpn Heart J 1993; 34: 403-17.

12. Shoucri RM. Possible clinical applications of the external work reverse of the myocardium. Jpn Heart J 1994; 35; $771-87$.

13. Shoucri RM. Clinical application of end-systolic pressure-volume relation. Ann Biomed Eng 1994; 22 : 212-7.

14. Shoucri RM. The active force of the myocardium, In: Power H, Hart RT, editors. Computer Simulations in Biomedicine. Boston: Computational Mechanics Publications, 1995: 19-26.

15. Elzinga $G$, Westerhof N. Matching between ventricle and arterial load, an evolutionary process. Circ Res 1991; 68: 1495-500.

16. Takaoka $\mathrm{H}$, Takeuchi $\mathbf{M}$, Odake $\mathrm{M}$, et al. Depressed contractile state and increased myocardial consumption for non-mechanical work in patients with heart failure due to old myocardial infarction. Cardiov Res 1994; 28: 1251-7.

17. Glower DD, Spratt JA, Snow ND, et al. Linearity of the Frank-Starling relationship in the intact heart: the concept of preload recruitable stroke work. Circulation 1985; 71; 994-1009.

18. Sunagawa K, Maughan WL, Sagawa K. Optimal arterial resistance for maximal stroke work studied in isolated canine left ventricle. Circ Res 1985; 56: 586-95.

19. Suga H. Paul Dudley White International Lecture: Cardiac performance as viewed through the pressure-volume window. Jpn Heart J. 35: 263-80. 